\title{
Análise da seleção de hiperparâmetros de Data Augmentation na detecção de Covid-19 em imagens de raio-x com Deep Learning
}

\author{
Pedro Rici, Samara Oliveira Silva Santos, André Luiz Carvalho Ottoni \\ Centro de Ciências Exatas e Tecnológicas \\ Universidade Federal do Recôncavo da Bahia \\ Cruz das Almas, Brasil \\ E-mail: pedrorici@ieee.org, oss.samara@gmail.com, andre.ottoni@ufrb.edu.br
}

\begin{abstract}
Resumo - A pandemia de Covid-19 foi declarada em 2020 pela Organização Mundial da Saúde. Um dos aspectos mais relevante dessa doença respiratória é o fato que a infecção causada pelo novo coronavírus possui uma alta taxa de disseminação. Assim, o diagnóstico rápido e preciso pode contribuir na redução da taxa de transmissão. Nesse aspecto, na literatura, técnicas de Deep Learning são estudadas para a aplicação na detecção dessa doença através de imagens de raio- $x$ do pulmão do paciente. No entanto, um dos desafios dessa área é o treinamento de modelos de Redes Neurais Convolucionais com base de dados com poucas amostras. Uma possibilidade é a geração de imagens artificiais através de técnicas de Data Augmentation. Dessa forma, o objetivo deste trabalho é propor uma metodologia criteriosa para seleção de hiperparâmetros de Data Augmentation para a classificação de imagens de raio-x de pulmão na detecção de Covid-19 com Deep Learning. A metodologia proposta consiste na análise de acurácia de 16 combinações de transformações (zoom, rotação, intensidade do brilho e espelhamento horizontal) aplicadas na geração de novas imagens para treinamento. Após a seleção dos hiperparâmetros, o sistema classificador alcançou acurácias de até $100 \%$ de acertos na etapa de teste.
\end{abstract}

Palavras-Chave-Deep learning, Data Augmentation, Covid-19, Rede Neural Convolucional, Imagens de Raio-x.

\section{INTRODUÇÃO}

Covid-19 é uma doença respiratória e infectuosa causado pelo novo coronavírus (SARS-CoV-2) [1]-4]. A nova doença foi identificada pela primeira vez em 2019 , em Wuhan na China e rapidamente se espalhou pelo mundo, até que no 11 de março de 2020, a Organização Mundial de Saúde (OMS) declarou Pandemia de Covid-19 11. Para identificar a contaminação por Covid-19, existem testes que aliados aos sintomas clínicos podem confirmar o diagnóstico 5. Uma forma de realizar a detecção é através de testes moleculares, quando se detecta a presença de material genético do vírus no paciente; ou sorológico, quando detecta-se os anticorpos 2 .

O diagnóstico rápido e preciso do vírus de Covid-19 auxilia a diminuir a taxa de transmissão e também realizar um acompanhamento do estado do paciente [6]. Porém a falta de testes, o tempo de espera dos resultados e o desgaste dos profissionais da saúde dificulta na agilidade do diagnóstico [7]. De forma, que alternativas para auxiliar na detecção do coronavírus estão surgindo para agilizar e diminuir o custo 7], 8. Uma dessas vertentes é o uso de Machine Learning para detecção de Covid-19 através de imagens de raio-x de pacientes com sintomas $[9]-15]$. Nessa linha, o trabalho de [16] desenvolve uma nova arquitetura para classificar imagens de raio-x em três grupos de pacientes: saudáveis, pneumonia viral, pneumonia Covid19. Outro exemplo, é o estudo de [13, que realiza uma extensa pesquisa bibliográfica e reúne os principais avanços na área na aplicação de Deep Learning no diagnóstico de Covid-19 em imagens.

No entanto, um dos desafios desse campo de estudo é a seleção das arquiteturas de métodos de Machine Learning e o ajuste dos hiperparâmetros de forma melhorar o desempenho na classificação das imagens 9]-12. Nessa linha, alguns trabalhos já realizaram estudos comparativos de diferentes arquiteturas de Deep Learning para a detecção de Covid-19 em imagens de raio-x 91, 11, [14. Nesse sentido, o trabalho de 9 analisa duas diferentes arquiteturas de Redes Neurais Convolucionais (ResNet50 e ResNet101) obtém uma acurácia de 97,77\% na detecção de Covid-19 em imagens de raio-x. O estudo de [11], por sua vez, compara cinco diferentes arquiteturas de Deep Learning (DenseNet, InceptionV3, MobileNet, ResNet50, VGG16 e VGG19), atingindo acurácia de $98,81 \%$ para a classificação binária e 91,68\% para a classificação multiclasse.

Seguindo essa vertente da definição de boas condições para experimentos com Deep Learning, uma importante área é o Data Augmentation [17-19]. Essa técnica é utilizada para reduzir o overfitting, gerando imagens artificiais de treinamento através de transformações aleatórias 20 . A aplicação dessa técnica torna-se importante principalmente nos casos em que o número de amostras para treinamento é relativamente pequeno 17], 20]. Nesse contexto, encontram-se boa parte dos estudos de aplicação de Deep Learning no diagnóstico de Covid-19 via imagens, tendo em vista realizarem experimentos com banco de dados com poucos exemplos [15], 21], 22]. Dentro desse aspecto, alguns trabalhos discorrem sobre o papel do Data Augmentation na classificação de Covid-19 em imagens de raio-x [15], 21], 22. No entanto, pouca atenção foi dada na literatura para a análise de hiperparâmetros (transformações nas imagens) 
de Data Augmentation quando se refere ao diagnóstico de Covid-19 utilizando imagens de raio-x 15.

O objetivo deste trabalho é propor uma metodologia experimental para a definição de hiperparâmetros de Data Augmentation na classificação de imagens de raio-x para detecção de Covid-19 com Deep Learning. Para isso, fazse necessário estudar e compreender o funcionamento das técnicas de Deep Learning, aplicar uma arquitetura de Machine Learning para esse tipo de classificação e analisar a acurácia para diferentes combinações de hiperparâmetros. Foi selecionada uma uma Rede Neura Convolucional abordada na literatura 23], 24], e assim realizada a classificação a partir de dois grupos experimentais: (1) entre imagens de raio-x de pacientes normais versus pacientes com Covid-19; (2) entre imagens de raio- $\mathrm{x}$ de pacientes com pneumonia versus pacientes com Covid-19.

O presente do trabalho está dividido em seis seções: A Seção II apresenta a fundamentação teórica. A Seção III apresenta o banco de dados, a arquitetura e hiperparâmetros e os métodos utilizados no trabalho. A Seção IV apresenta os resultados obtidos. A Seção V apresenta uma comparação entre os resultados obtidos nesse trabalho e com outros trabalhos relacionados. Por fim, a Seção VI apresenta as contribuições feitas por esse trabalho, além de sugestões para futuros trabalhos.

\section{FundamentaÇÃo TEÓRICA}

Redes Neurais Artificiais (RNAs) são métodos de aprendizado de máquina supervisionado amplamente abordados na literatura [25]-27]. Nesse aspecto, as RNAs possuem diversas aplicações como em reconhecimento facial 28, [29], bolsa de valores [30], 31] e carros autônomos 32], 33 .

A inspiração para a criação da RNA surgiu com o conceito do sistema nervoso humano [25], onde há semelhanças entre as estruturas físicas e funções do sistema nervoso com o neurônio artificial. Um exemplo de RNA pode ser representado pela Figura 1 .

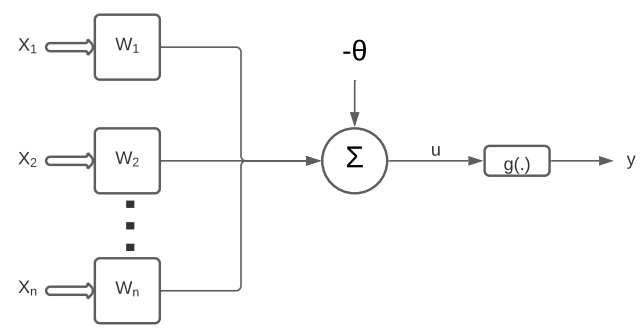

Figura 1: Neurônio Artificial. Baseado em 25.

onde $x_{1}, x_{2}$ e $x_{n}$ são os sinais de entrada, $w_{1}, w_{2}$ e $w_{n}$ são os pesos sinápticos, $\sum$ é o combinador linear, $\theta$ é o limiar de ativação, $u$ é o potencial de ativação, $g$ é a função de ativação, e por fim $y$ é o sinal de saída. As Equações (1) e (2) resumem o resultado produzido pelo neurônio artificial.

$$
\begin{array}{r}
u=\sum_{i=1}^{n} w_{i} \cdot x_{i}-\theta \\
y=g(u)
\end{array}
$$

As Redes Neurais Convolucionais, do inglês Convolutional Neural Network (CNN), são um tipo de RNA. Uma CNN tem como característica a sua eficiência e robustez em tratar as informações a partir de um grande número de camadas e neurônios 23], [25], 34], 35].

A CNN possui 4 operações básicas [23], 34], 35]:

1) Operador de Convolução (Kernel): A matriz de convolução é multiplicada pela matriz imagem para aplicar os efeitos de imagem. O resultado dessa multiplicação é um mapa de características, onde são armazenadas as características mais importantes, diminuindo o tamanho da matriz original para facilitar o processamento. Depois de obtido o mapa de características, em geral, é aplicada a função de ativação Relu.

2) Pooling: É a camada responsável para enfatizar as características importantes resultantes da aplicação da função Relu, reduzindo o overfitting e ruídos desnecessários.

3) Flattening: É a camada que transforma a matriz resultante do Pooling em um vetor, podendo assim iniciar a aplicação na rede neural densa.

4) Rede Neural Densa: É a rede com os neurônios semelhantes a rede formada pela RNA tradicional, possuem conjuntos de neurônios e camadas totalmente conectados que em conjunto define as classes de saída.

Para auxiliar no desenvolvimento de uma CNN podem ser utilizadas bibliotecas em linguagem de programação. Um exemplo é o Keras, uma biblioteca baseada no TensorFlow, gratuita e disponível em Python ou R [20], 23.

\section{MetodologiA}

A metodologia proposta neste trabalho é composta por cinco etapas: (1) o banco de dados utilizado, (2) o préprocessamento de imagens, (3) a RNA utilizada, (4) a fase de treinamento e validação, e por fim (5) o teste.

\section{A. Base de dados}

A base de imagens selecionada está disponibilizada na plataforma Kaggle ${ }^{1}$ Esse banco de dados contém imagens obtidas de três diferentes fontes $2^{2}$ De acordo com as informações disponibilizadas na página do Kaggle, todas as fontes possuem licença livre e podem ser utilizados para fins de pesquisa, avaliação e comercial.

${ }_{1}^{1}$ https://www.kaggle.com/prashant268/ chest-xray-covid19-pneumonia

2 https://github.com/agchung

3 https://www.kaggle.com/paultimothymooney/ chest-xray-pneumonia

${ }^{4}$ https://github.com/ieee8023/covid-chestxray-dataset 
O banco de dados possui no total 6432 imagens, divididas em três classes:

- Covid-19: 576 imagens pulmonares de raio-x de pacientes diagnosticados com Covid-19.

- Pneumonia: 4273 imagens pulmonares de raio-x de pacientes diagnosticados com pneumonia.

- Normal: 1583 imagens pulmonares de raio-x de pacientes diagnosticados sem Covid-19 e pneumonia.

A Figura 2 demonstra alguns exemplos de imagens disponibilizadas no banco de dados.

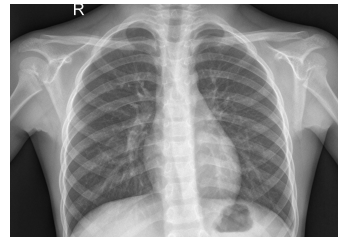

(a) Classe Normal.

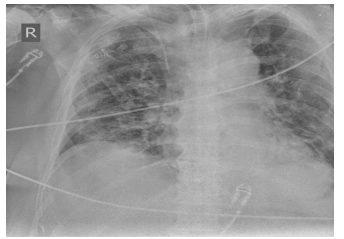

(c) Classe Covid-19.

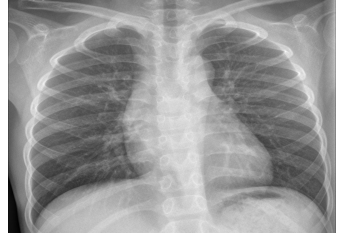

(b) Classe Normal.

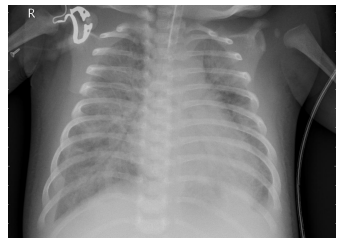

(d) Classe Pneumonia.
Figura 2: Exemplo de imagens, para cada classe (Normal, Covid-19 e Pneumonia) disponibilizadas no banco de dados.

\section{B. Pré-Processamento}

Após a definição da base de dados, as imagens foram reorganizadas em dois grupos experimentais, Covid-19 versus Pneumonia e Covid-19 versus Normal, e separadas em três conjuntos (treino, validação e teste) do seguinte modo:

- Covid-19 versus Pneumonia

- Treinamento: 432 imagens da classe Covid-19 e 432 imagens da classe Pneumonia.

- Validação: 72 imagens da classe Covid-19 e 72 imagens da classe Pneumonia.

- Teste: 72 imagens da classe Covid-19 e 72 imagens da classe Pneumonia.

- Covid-19 versus Normal

- Treinamento: 432 imagens da classe Covid-19 e 432 imagens da classe Normal.

- Validação: 72 imagens da classe Covid-19 e 72 imagens da classe Normal.

- Teste: 72 imagens da classe Covid-19 e 72 imagens da classe Normal.

Dessa forma, o banco de dados reorganizado passou a contar com 1728 imagens. Além disso, as imagens foram divididas em $75 \%$ para treinamento, $12,5 \%$ para validação e $12,5 \%$ para teste.
A quantidade de imagens contidas no banco de dados é consideravelmente pequena. Dessa forma, metodologia proposta visa aplicar a técnica de Data Augmentation [17], 20 para gerar novas imagens e consequentemente aumentar a quantidade de dados para treinamento. Para isso, foi adotada a função ImageDataGenerator() disponibilizada pela biblioteca Keras 20], onde essa gera novas imagens, conforme a necessidade, de modo online, ou seja, os novos lotes de dados de imagens são gerados em tempo real.

A metodologia proposta selecionou quatro transformações de Data Augmentation para serem analisadas:

- rotation range: representado por um valor inteiro que identifica o valor máximo de rotação que a imagem pode possuir

- brightness range: uma lista ou tupla que representa a faixa de brilho que a imagem pode apresentar

- zoom range: um valor do tipo float ou um vetor [mín, máx] que determina a faixa de zoom que a imagem pode obter

- horizontal flip: do tipo boleano onde a imagem pode ser invertida horizontalmente ou não.

Assim, neste estudo foram definidos dois níveis experimentais para análise desses hiperparâmetros de Data Augmentation:

- rotation_range: 60 e 140;

- brightness_range: $[0.3,1.0]$ e $[0.2,0.8]$;

- zoom_range: $[0.3,1.0]$ e $[0.2,0.8]$;

- horizontal_flip: True e False.

A Figura 3 exemplifica algumas imagens geradas a partir da utilização da função ImageDataGenerator () com os seguintes valores de hiperparâmetros:

- rotation_range: 60 ;

- brightness_range: $[0.2,0.8]$;

- zoom_range: $[0.3,1.0]$.

- horizontal_flip: True.

\section{Arquitetura da Rede Neural Convolucional}

A arquitetura de CNN utilizada nesse trabalho foi proposta por 23. Essa arquitetura possui 12 camadas, sendo quatro convolucionais, quatro de Max Pooling, uma camada Flatten, uma de Dropout, uma camada densa com 512 neurônios e a camada de saída com o neurônio classificador. A arquitetura utilizada pode ser representada pela Figura 4 .

Além dos hiperparâmetros de data augmentation citados anteriormente, foram utilizados hiperparâmetros fixos para as etapas experimentais da $\mathrm{CNN}$ : número de filtros em cada camada convolucional $(32,64,128,128)$, kernel size nas camadas convolucionais $(3 \times 3)$, pool size nas camadas de Max Pooling $(2 \times 2)$ e funções de ativação $R E L U$ nas camadas convolucionais/densa e sigmoid da camada de classificação. A arquitetura da $\mathrm{CNN}$ foi codificadas a partir do comando keras.models.Sequential(), disponível da biblioteca Keras da linguagem Python 23. Também definida a taxa de dropout em 0,2, utilizadas as funções 


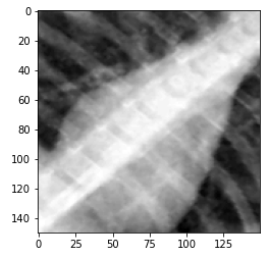

(a) Classe Normal.

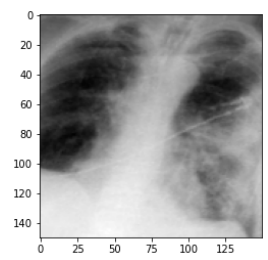

(c) Classe Covid-19.

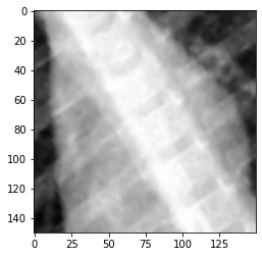

(b) Classe Normal.

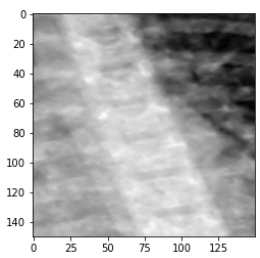

(d) Classe Pneumonia.
Figura 3: Exemplos de imagens geradas com data augmentation para as classe Normal, Pneumonia e Covid-19.

de ativação Relu e Sigmoid, e o otimizador Adagrad com taxa de aprendizado de 0,001.

\section{Treinamento e Validação}

Os experimentos de treinamento e validação visam avaliar as combinações de hiperparâmetros de Data Augmentation. Nesse aspecto, os passos propostos pela metodologia proposta neste estudo são representados na Figura 5.

A Tabela I] por sua vez, apresenta as 16 combinações de hiperparâmetos analisadas.

Tabela I: Combinações de hiperparâmetros de Data Augmentation utilizadas.

\begin{tabular}{|c|c|c|c|c|}
\hline Combinação & Rotation & Brightness & Zoom & Horizontal Flip \\
\hline 1 & 60 & {$\left[\begin{array}{lll}0.3 & 1.0\end{array}\right]$} & {$\left[\begin{array}{lll}0.3 & 1.0\end{array}\right]$} & True \\
\hline 2 & 60 & {$\left[\begin{array}{ll}0.2 & 0.8\end{array}\right]$} & {$\left[\begin{array}{ll}0.3 & 1.0\end{array}\right]$} & True \\
\hline 3 & 60 & $0.31 .0]$ & {$\left[\begin{array}{ll}0.2 & 0.8\end{array}\right]$} & True \\
\hline 4 & 60 & {$\left[\begin{array}{ll}0.2 & 0.8\end{array}\right]$} & {$\left[\begin{array}{ll}0.2 & 0.8\end{array}\right]$} & True \\
\hline 5 & 60 & {$\left[\begin{array}{ll}0.3 & 1.0\end{array}\right]$} & {$\left[\begin{array}{ll}0.3 & 1.0\end{array}\right]$} & False \\
\hline 6 & 60 & {$\left[\begin{array}{ll}0.2 & 0.8\end{array}\right]$} & {$\left[\begin{array}{ll}0.3 & 1.0\end{array}\right]$} & False \\
\hline 7 & 60 & {$\left[\begin{array}{ll}0.3 & 1.0\end{array}\right]$} & {$\left[\begin{array}{ll}0.2 & 0.8\end{array}\right]$} & False \\
\hline 8 & 60 & {$\left[\begin{array}{ll}0.2 & 0.8\end{array}\right]$} & {$\left[\begin{array}{ll}0.2 & 0.8\end{array}\right]$} & False \\
\hline 9 & 140 & {$\left[\begin{array}{ll}0.3 & 1.0\end{array}\right]$} & {$\left[\begin{array}{ll}0.3 & 1.0\end{array}\right]$} & True \\
\hline 10 & 140 & {$\left[\begin{array}{ll}0.2 & 0.8\end{array}\right]$} & {$\left[\begin{array}{ll}0.3 & 1.0\end{array}\right]$} & True \\
\hline 11 & 140 & {$\left[\begin{array}{ll}0.3 & 1.0\end{array}\right]$} & {$\left[\begin{array}{ll}0.2 & 0.8\end{array}\right]$} & True \\
\hline 12 & 140 & {$\left[\begin{array}{ll}0.2 & 0.8\end{array}\right]$} & {$\left[\begin{array}{ll}0.2 & 0.8\end{array}\right]$} & True \\
\hline 13 & 140 & {$\left[\begin{array}{ll}0.3 & 1.0\end{array}\right]$} & {$\left[\begin{array}{ll}0.3 & 1.0\end{array}\right]$} & False \\
\hline 14 & 140 & {$\left[\begin{array}{ll}0.2 & 0.8\end{array}\right]$} & {$\left[\begin{array}{ll}0.3 & 1.0\end{array}\right]$} & False \\
\hline 15 & 140 & {$\left[\begin{array}{ll}0.3 & 1.0\end{array}\right]$} & {$\left[\begin{array}{ll}0.2 & 0.8\end{array}\right]$} & False \\
\hline 16 & 140 & {$\left[\begin{array}{ll}0.2 & 0.8\end{array}\right]$} & {$\left[\begin{array}{ll}0.2 & 0.8\end{array}\right]$} & False \\
\hline
\end{tabular}

Nesse sentido, cada uma das combinações apresentadas na Tabela 1 foi simuladas em 3 repetições com 10 épocas. Além disso, para o treinamento dos modelos de CNN foi utilizada a função fit_generator() da biblioteca Keras [23.

$\mathrm{Na}$ etapa de validação, por sua vez, foi realizada a análise de desempenho da CNN para cada combinação de transformação Data Augmentation. Essa avaliação foi realizada a partir da observação da métrica de acurácia na classificação das imagens, conforme Eq. (3):

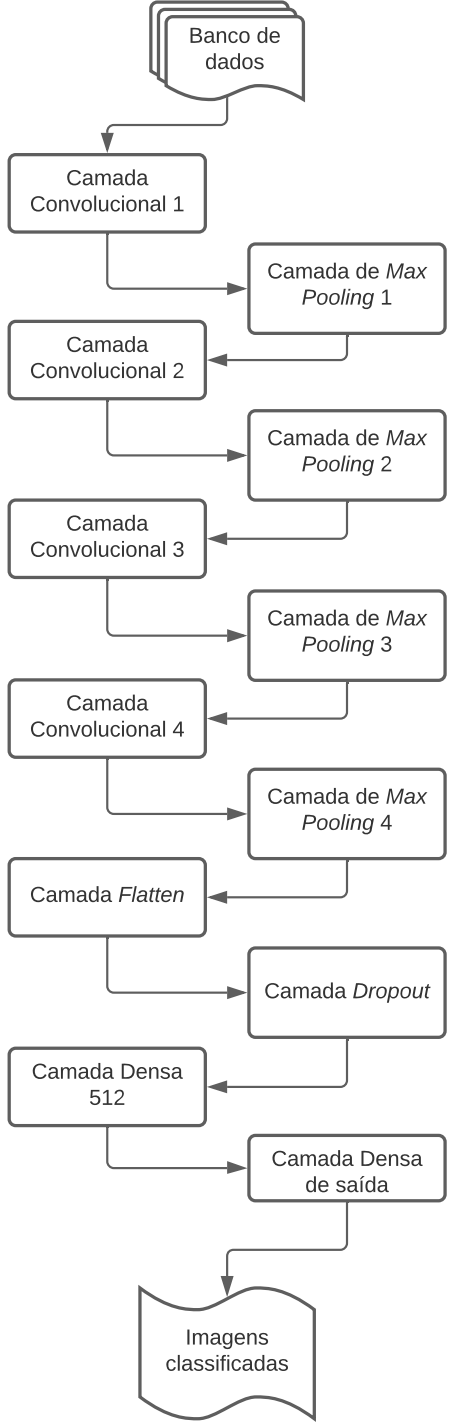

Figura 4: Fluxograma para representação da Rede Neural Convolucional CNN-12 adotada no trabalho. Baseada em 23 e 24.

$$
A_{c}=\frac{V_{P}+V_{N}}{V_{P}+V_{N}+F_{P}+F_{N}}
$$

em que, $A_{c}$ é o valor da acurácia, $V_{P}$ são os verdadeiros positivos, $V_{N}$ são os verdadeiros negativos, $F_{P}$ são os falsos positivos e $F_{N}$ são os falsos negativos.

\section{E. Teste}

Na sequência da metodologia proposta, para a realização da etapa de teste foram selecionadas as três combinações de hiperparâmetros que obtiveram com os melhores valores de acurácia média na etapa de validação. Nesta fase, as imagens analisadas são distintas das etapas de treinamento e validação, conforme divisão do banco de dados descrita na SeçãoIII-B. 


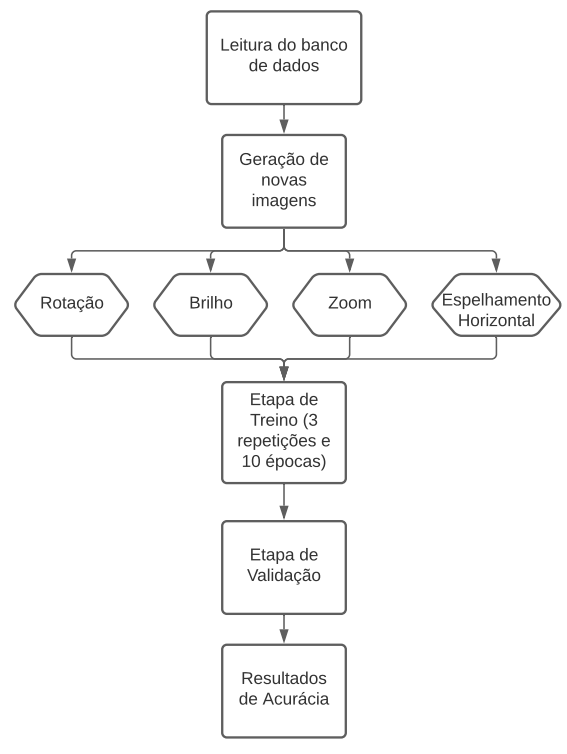

Figura 5: Fluxograma da metodologia para seleção de hiperparâmetros de Data Augmentation - treinamento e validação.

A arquitetura CNN foi mantida e para cada combinação de hiperparâmetros foram realizadas 3 repetições com 30 épocas. Portanto, o objetivo da etapa de teste é selecionar qual combinação de hiperparâmetros resulta na melhor acurácia quando analisado um novo conjunto de imagens. Para isso, foi utilizado o o comando evaluate_generator() da biblioteca Keras para a avaliação da acurácia na etapa de teste 23].

\section{Resultados}

Nesta seção, são apresentados os resultados referentes às seções [III-D e III-E nas fases de treinamento, validação e teste da aplicação da Rede Neural Convolucional. Em cada uma das etapas foram selecionadas as combinações de hiperparâmetros de Data Augmentation com as melhores métricas de acurácia para classificação de imagens nos dois grupos experimentais: (1) Normal versus Covid-19 e (2) Pneumonia versus Covid-19.

\section{A. Treinamento e Validação}

A Tabela II apresenta os valores de acurácia para no processo de classificação do grupo experimental Normal versus Covid-19.

A partir da Tabela II é possível observar a influência dos hiperparâmetros de data augmentation nos valores de acurácia. Nesse sentindo, destaca-se as acurácias média de $91,7 \%, 90,6 \%$ e $86,5 \%$ como os melhores resultados obtidos, respectivamente pelas combinações 2, 5 e 6 . Essas três combinações com maiores resultados médios de acurácia resultados foram selecionadas para a etapa de teste do grupo experimental Normal $\times$ Covid-19. É importante pontuar que as transformação de rotação de $60^{\circ}$ e o zoom
Tabela II: Resultados de Acurácia (\%) para Validação na Etapa 1 de Combinações de Data Augmentation para Normal $\times$ Covid-19.

\begin{tabular}{|c|c|c|c|c|c|c|c|}
\hline Rot. & Brig. & Zoom & Hor. Flip & $A c_{1}(\%)$ & $A c_{2}(\%)$ & $A c_{3}(\%)$ & Med.(\%) \\
\hline 60 & {$\left[\begin{array}{ll}0.3 & 1.0\end{array}\right]$} & $\left.\begin{array}{|ll}0.3 & 1.0\end{array}\right]$ & True & 78,1 & 43,8 & 75,0 & 65,6 \\
\hline 60 & {$\left[\begin{array}{ll}0.2 & 0.8\end{array}\right]$} & {$\left[\begin{array}{ll}0.3 & 1.0\end{array}\right]$} & True & 90,6 & 96,9 & 84,4 & 90,6 \\
\hline 60 & {$\left[\begin{array}{ll}0.3 & 1.0\end{array}\right]$} & {$\left[\begin{array}{ll}0.2 & 0.8\end{array}\right]$} & True & 71,9 & 43,8 & 43,8 & 53,1 \\
\hline 60 & {$\left[\begin{array}{ll}0.2 & 0.8\end{array}\right]$} & {$\left[\begin{array}{ll}0.2 & 0.8\end{array}\right]$} & True & 84,4 & 68,8 & 65,6 & 72,9 \\
\hline 60 & {$\left[\begin{array}{ll}0.3 & 1.0\end{array}\right]$} & {$\left[\begin{array}{ll}0.3 & 1.0\end{array}\right]$} & False & 96,9 & 81,3 & 96,9 & 91,7 \\
\hline 60 & {$\left[\begin{array}{ll}0.2 & 0.8\end{array}\right]$} & {$\left[\begin{array}{ll}0.3 & 1.0\end{array}\right]$} & False & 90,6 & 90,6 & 78,1 & 86,5 \\
\hline 60 & {$\left[\begin{array}{ll}0.3 & 1.0\end{array}\right]$} & {$\left[\begin{array}{ll}0.2 & 0.8\end{array}\right]$} & False & 50,0 & 81,3 & 56,3 & 62,5 \\
\hline 60 & {$\left[\begin{array}{ll}0.2 & 0.8\end{array}\right]$} & {$\left[\begin{array}{ll}0.2 & 0.8\end{array}\right]$} & False & 71,9 & 78,1 & 90,6 & 80,2 \\
\hline 140 & {$\left[\begin{array}{ll}0.3 & 1.0\end{array}\right]$} & {$\left[\begin{array}{ll}0.3 & 1.0\end{array}\right]$} & True & 59,4 & 53,1 & 90,6 & 67,7 \\
\hline 140 & {$\left[\begin{array}{ll}0.2 & 0.8\end{array}\right]$} & {$\left[\begin{array}{ll}0.3 & 1.0\end{array}\right]$} & True & 31,3 & 75,0 & 53,1 & 53,1 \\
\hline 140 & {$\left[\begin{array}{ll}0.3 & 1.0\end{array}\right]$} & {$\left[\begin{array}{ll}0.2 & 0.8\end{array}\right]$} & True & 56,3 & 81,3 & 59,4 & 65,6 \\
\hline 140 & {$\left[\begin{array}{ll}0.2 & 0.8\end{array}\right]$} & {$\left[\begin{array}{ll}0.2 & 0.8\end{array}\right]$} & True & 40,6 & 50,0 & 43,8 & 44,8 \\
\hline 140 & {$\left[\begin{array}{ll}0.3 & 1.0\end{array}\right]$} & {$\left[\begin{array}{ll}0.3 & 1.0\end{array}\right]$} & False & 62,5 & 65,6 & 46,9 & 58,3 \\
\hline 140 & {$\left[\begin{array}{ll}0.2 & 0.8\end{array}\right]$} & {$\left[\begin{array}{ll}0.3 & 1.0\end{array}\right]$} & False & 62,5 & 87,5 & 71,9 & 74,0 \\
\hline 140 & {$\left[\begin{array}{ll}0.3 & 1.0\end{array}\right]$} & {$\left[\begin{array}{ll}0.2 & 0.8\end{array}\right]$} & False & 40,6 & 62,5 & 75,0 & 59,4 \\
\hline 140 & {$\left[\begin{array}{ll}0.2 & 0.8\end{array}\right]$} & {$\left[\begin{array}{ll}0.2 & 0.8\end{array}\right]$} & False & 65,6 & 84,4 & 59,4 & 69,8 \\
\hline
\end{tabular}

para uma faixa entre $30 \%$ e $100 \%$ estão presentes nos três melhores resultados de acurácia. Vale ressaltar também os piores desempenhos (baixa acurácia), como é o caso de $44,8 \%$ para a combinação 12 e $53,1 \%$ para as combinações 10 e 3.

Na Tabela III apresenta-se os resultados de acurácia referentes à primeira etapa da metodologia para a classificação do grupo experimental Pneumonia versus Covid-19.

Tabela III: Resultados de Acurácia (\%) para Validação na Etapa 1 de Combinações de Data Augmentation para Pneumonia $\times$ Covid-19.

\begin{tabular}{|c|c|c|c|c|c|c|c|}
\hline Rot. & Brig. & Zoom & Hor. Flip & $A c_{1}(\%)$ & $A c_{2}(\%)$ & $A c_{3}(\%)$ & Méd.(\%) \\
\hline 60 & {$\left[\begin{array}{ll}0.3 & 1.0\end{array}\right]$} & {$\left[\begin{array}{ll}0.3 & 1.0\end{array}\right]$} & True & 90,6 & 84,4 & 90,6 & 88,5 \\
\hline 60 & {$\left[\begin{array}{ll}0.2 & 0.8\end{array}\right]$} & {$\left[\begin{array}{ll}0.3 & 1.0\end{array}\right]$} & True & 75,0 & 84,4 & 78,1 & 79,2 \\
\hline 60 & {$\left[\begin{array}{ll}0.3 & 1.0\end{array}\right]$} & [0.2 0.8 . & True & 68,8 & 59,4 & 71,9 & 66,7 \\
\hline 60 & {$\left[\begin{array}{ll}0.2 & 0.8\end{array}\right]$} & {$\left[\begin{array}{ll}0.2 & 0.8\end{array}\right]$} & True & 78,1 & 68,8 & 71,9 & 72,9 \\
\hline 60 & {$\left[\begin{array}{ll}0.3 & 1.0\end{array}\right]$} & {$\left[\begin{array}{ll}0.3 & 1.0\end{array}\right]$} & False & 84,4 & 81,3 & 62,5 & 76,0 \\
\hline 60 & {$\left[\begin{array}{ll}0.2 & 0.8\end{array}\right]$} & {$\left[\begin{array}{ll}0.3 & 1.0\end{array}\right]$} & False & 87,5 & 90,6 & 84,4 & 87,5 \\
\hline 60 & {$\left[\begin{array}{ll}0.3 & 1.0\end{array}\right]$} & {$\left[\begin{array}{ll}0.2 & 0.8\end{array}\right]$} & False & 84,4 & 78,1 & 81,3 & 81,3 \\
\hline 60 & {$\left[\begin{array}{ll}0.2 & 0.8\end{array}\right]$} & {$\left[\begin{array}{ll}0.2 & 0.8\end{array}\right]$} & False & 62,5 & 65,6 & 62,5 & 63,5 \\
\hline 140 & {$\left[\begin{array}{ll}0.3 & 1.0\end{array}\right]$} & {$\left[\begin{array}{ll}0.3 & 1.0\end{array}\right]$} & True & 81,3 & 65,6 & 71,9 & 72,9 \\
\hline 140 & {$\left[\begin{array}{ll}0.2 & 0.8\end{array}\right]$} & {$\left[\begin{array}{ll}0.3 & 1.0\end{array}\right]$} & True & 81,3 & 71,9 & 65,6 & 72,9 \\
\hline 140 & {$\left[\begin{array}{ll}0.3 & 1.0\end{array}\right]$} & {$\left[\begin{array}{ll}0.2 & 0.8\end{array}\right]$} & True & 59,4 & 59,4 & 62,5 & 60,4 \\
\hline 140 & {$\left[\begin{array}{ll}0.2 & 0.8\end{array}\right]$} & {$\left[\begin{array}{ll}0.2 & 0.8\end{array}\right]$} & True & 46,9 & 65,6 & 53,1 & 55,2 \\
\hline 140 & {$\left[\begin{array}{ll}0.3 & 1.0\end{array}\right]$} & [0.3 1.0] & False & 50,0 & 71,9 & 59,4 & 60,4 \\
\hline 140 & {$\left[\begin{array}{ll}0.2 & 0.8\end{array}\right]$} & {$\left[\begin{array}{ll}0.3 & 1.0\end{array}\right]$} & False & 56,3 & 81,3 & 78,1 & 71,9 \\
\hline 140 & {$\left[\begin{array}{ll}0.3 & 1.0\end{array}\right]$} & {$\left[\begin{array}{ll}0.2 & 0.8\end{array}\right]$} & False & 59,4 & 59,4 & 50,0 & 56,3 \\
\hline 140 & {$\left[\begin{array}{ll}0.2 & 0.8\end{array}\right]$} & {$\left[\begin{array}{ll}0.2 & 0.8\end{array}\right]$} & False & 75,0 & 46,9 & 62,5 & 61,5 \\
\hline
\end{tabular}

Rot.: rotação; Brig.: brilho; Hor. Flip: espelhamento horizontal.

A partir da Tabela III também pode-se notar que os hiperparâmetros de Data Augmentation interferem diretamente no desempenho da CNN. Por exemplo, o valor médio de acurácia variaram entre $55,2 \%$ e $88,5 \%$. Destaca-se que as combinações que alcançaram maiores acurácias médias foram: combinação 1 (88,5\%); combinação $6(87,5 \%)$; e a combinação 7 (81,3\%). Assim, essas 3 combinações foram selecionadas para a etapa de testes dos experimentos de Pneumonia $\times$ Covid -19 .

\section{B. Teste}

A Tabela IV apresenta os resultados de acurácia referentes a segunda etapa da metodologia para a classificação do grupo experimentação Normal versus Covid-19. 
Tabela IV: Resultados de Acurácia (\%) para Teste na Etapa 2 de Combinações de Data Augmentation para Normal $\times$ Covid-19.

\begin{tabular}{cccccccc}
\hline Rot. & Brig. & Zoom & Hor. Flip & $A c_{1}(\%)$ & $A c_{2}(\%)$ & $A c_{3}(\%)$ & Méd.(\%) \\
\hline 60 & {$\left[\begin{array}{lll}0.3 & 1.0\end{array}\right]$} & {$\left[\begin{array}{lll}0.3 & 1.0\end{array}\right]$} & False & 96,9 & 93,7 & 96,9 & $\mathbf{9 5 , 8}$ \\
60 & {$\left[\begin{array}{ll}0.2 & 0.8\end{array}\right]$} & {$\left[\begin{array}{lll}0.3 & 1.0\end{array}\right]$} & True & 93,7 & 90,6 & $\mathbf{1 0 0 , 0}$ & 94,8 \\
60 & {$\left[\begin{array}{ll}0.2 & 0.8\end{array}\right]$} & {$\left[\begin{array}{lll}0.3 & 1.0\end{array}\right]$} & False & 90,6 & 93,7 & 90,6 & 91,7 \\
\hline
\end{tabular}

Pode-se citar novamente a influência dos hiperparâmetros, onde na etapa de teste para as classes Normal versus Covid-19 foi obtido um valor médio máximo de 95,8\% para a combinação 5 e um valor médio mínimo de $91,7 \%$ para a combinação 6. Vale destacar também que foi obtido uma acurácia de $100 \%$ para um dos testes, para a combinação 2.

A Tabela V apresenta os resultados de acurácia referentes à segunda etapa da metodologia para a classificação Pneumonia versus Covid-19. Nela temos a acurácia para as 3 melhores combinações de hiperparâmetros de Data Augmentation obtidos na etapa 1.

Tabela V: Resultados de Acurácia (\%) para Teste na Etapa 2 de Combinações de Data Augmentation para Pneumonia $\times$ Covid-19

\begin{tabular}{|c|c|c|c|c|c|c|c|}
\hline Rot. & Brig. & Zoom & Hor. Flip & $A c_{1}(\%)$ & $A c_{2}(\%)$ & $A c_{3}(\%)$ & Méd.(\%) \\
\hline 60 & {$\left[\begin{array}{ll}0.3 & 1.0\end{array}\right]$} & {$\left[\begin{array}{ll}0.3 & 1.0\end{array}\right]$} & True & 90,6 & 78,1 & 100,0 & 89,6 \\
\hline 60 & {$\left[\begin{array}{ll}0.2 & 0.8\end{array}\right]$} & {$\left[\begin{array}{ll}0.3 & 1.0\end{array}\right]$} & False & 87,5 & 84,4 & 87,5 & 86,5 \\
\hline 60 & {$\left[\begin{array}{ll}0.3 & 1.0\end{array}\right]$} & {$\left[\begin{array}{ll}0.2 & 0.8\end{array}\right]$} & False & 84,4 & 81,3 & 87,5 & 84,4 \\
\hline
\end{tabular}

Novamente, os experimentos revelaram a importância dos hiperparâmetros de Data Augmentation nos resultados de uma CNN. Nota-se que a combinação 1 apresentou o melhor valor de acurácia na média, com cerca de 89,6\% e também obteve acurácia máxima de 100\%.

\section{ComparaÇÃo COM OUtros trabalhos}

Nessa seção, a tabela VI apresenta a comparação entre a presente proposta com outros trabalhos que utilizam Deep Learning na classificação de Covid-19 em imagens de raio-x de pulmão: I [9], II [10 e III 36].

A Tabela VI revela que os estudos analisados dedicamse a análise de como a mudança de arquitetura da CNN interfere na acurácia. Por outro lado, a proposta deste trabalho visa analisar a influência dos hiperparâmetros de Data Augmentation. Nesse sentido, uma importante contribuição deste trabalho é a aplicação de Deep Learning na classificação de imagens de raio-x de pulmão para diagnóstico de Covid-19, considerando a influência dos hiperparâmetros de Data Augmentation.

Também vale destacar que a presente proposta adota uma metodologia para selecionar os hiperparâmetros de Data Augmentation relevantes nas etapas de treinamento e validação de um modelo CNN : rotation, brightness, zoom e horizontal flip. Os demais estudos analisados na Tabela VI utilizam apenas um ou dois desses hiperparâmetros.

Destaca-se também o uso da biblioteca Keras, que é utilizado no presente trabalho e na maioria dos trabalhos analisados na Tabela $\mathrm{VI}$, com exceção do trabalho I, que utiliza da biblioteca FastAi.

Por fim, destaca-se que todos os trabalhos analisados, inclusive a proposta apresentada, utiliza as mesmas classificações para as imagens: Normal, Pneumonia e Covid-19. Além disso, o modelo de CNN selecionado alcançou acurácia igual a $100 \%$ para os dois grupos de experimentos na etapa de teste. Esse patamar de taxa de acertos $(=100 \%)$ apenas foi apresentado nos resultados de um dos estudos analisado: [9]. Assim, indicando um bom ajuste do modelo proposto na tarefa de reconhecer Covid-19 em imagens de raio-x de pulmão.

Tabela VI: Comparação entre a presente proposta (Prop.) com trabalhos que utilizam Deep Learning na classificação de Covid-19 em imagens de raio-x de pulmão: I 9], II [10 e III 36 .

\begin{tabular}{cccccc}
\hline & & Prop. & I & II & III \\
\hline \multirow{2}{*}{ Metodologia } & Influência do & $\checkmark$ & - & - & - \\
& Data Augmentation & $\checkmark$ & & & \\
& Influência da & & & & \\
& arquitetura & - & $\checkmark$ & $\checkmark$ & $\checkmark$ \\
\hline \multirow{2}{*}{ Hiperparâmetros de } & Rotation & $\checkmark$ & $\checkmark$ & $\checkmark$ & $\checkmark$ \\
Data Augmentation & Brightness & $\checkmark$ & - & - & - \\
& Zoom & $\checkmark$ & - & $\checkmark$ & - \\
& Horizontal Flip & $\checkmark$ & - & - & $\checkmark$ \\
\hline \multirow{2}{*}{ Biblioteca } & Keras & $\checkmark$ & - & $\checkmark$ & $\checkmark$ \\
& FastAi & - & $\checkmark$ & - & - \\
\hline \multirow{2}{*}{ Classes de imagem } & Normal & $\checkmark$ & $\checkmark$ & $\checkmark$ & $\checkmark$ \\
& Pneumonia & $\checkmark$ & $\checkmark$ & $\checkmark$ & $\checkmark$ \\
\hline Acurácia máx & Covid-19 & $\checkmark$ & $\checkmark$ & $\checkmark$ & $\checkmark$ \\
\hline
\end{tabular}

\section{Conclusão}

O objetivo deste trabalho foi propor uma metodologia para seleção de hiperparâmetros de Data Augmentation na classificação de imagens de raio-x de pulmão. Assim, o presente estudo concentra suas contribuições nos seguintes aspectos: i) aplicação de modelo de Deep Learning no processo de detecção de Covid-19 através de imagens de raio-x de pulmão em bases de dados com poucas amostras; ii) proposta de uma metodologia de seleção de hiperparâmetros de Data Augmentation para essa aplicação; iii) acurácia máxima igual a 100\% na etapa de teste após o ajuste dos hiperparâmetros.

Os resultados encontrados demonstram a influência que os hiperparâmetros de Data Augmentation possuem na acurácia da classificação de imagens de raio-x de: i) pacientes saudáveis, ii) pacientes com pneumonia e iii) pacientes com Covid-19. Com a variação de 4 hiperparâmetros e um total de 16 combinações, foi obtido valores de acurácia entre $53,1 \%$ e $91,7 \%$ para a classificação Normal versus Covid-19 e entre $55,2 \%$ e 88,5\% para a classificação Pneumonia versus Covid-19, para a etapa de validação. Após a escolha das três combinações com maior acurácia e a realização da etapa de teste, os resultados de acurácia média aumentaram, chegando a atingir 95,8\% para a classificação Normal versus Covid-19 e 89,6\% para a classificação Pneumonia versus Covid-19. Vale destacar que em ambas as classificações 
foi ainda possível obter valores de acurácia de 100\%, demostrando a eficácia dos hiperparâmetros escolhidos para essa aplicação.

Para futuros trabalhos, sugere-se aplicar a análise para outros modelos de Redes Neurais Convolucionais. Assim como analisar a influência de outros hiperparâmetros de Data Augmentation nesse tipo de classificação e analisar como os mesmos influenciam individualmente nos resultados de acurácia.

\section{REFERÊNCIAS}

[1] A. Moreira and L. Pinheiro, "Oms declara pandemia de coronavírus," 2020. [Online]. Available: https://g1.globo.com/bemestar/coronavirus/noticia/2020/ 03/11/oms-declara-pandemia-de-coronavirus.ghtml

[2] D. B. Ribeiro, R. A. X. Ferreira, A. B. L. Marins, P. J. B da Silveira Júnior, and A. C. P. de Mello Pinheiro, "Covid-19: Um esclarecimento frente aos métodos diagnósticos," Revista Transformar, vol. 14, no. 2, pp. 205-215, 2020.

[3] T. Singhal, "A review of coronavirus disease-2019 (covid-19)," The indian journal of pediatrics, vol. 87, no. 4, pp. 281-286, 2020

[4] A. S. R. Souza, M. M. R. Amorim, A. S. d. O. Melo, A. M. Delgado, A. C. M. C. d. C. Florêncio, T. V. d. Oliveira, L. C. S. Lira, L. M. d. S. Sales, G. A. Souza, B. C. P. d. Melo et al., "Aspectos gerais da pandemia de covid-19," Revista Brasileira de Saúde Materno Infantil, vol. 21, pp. 29-45, 2021.

[5] V. d. C. Dias, M. Carneiro, L. Michelin, C. Vidal, L. Costa, C. Ferreira et al., "Testes sorológicos para covid-19: interpretação e aplicações práticas," J. Infect. Control, vol. 9, 2020.

[6] L. M. F. Vieira, E. Emery, and A. Andriolo, "Covid-19: laboratory diagnosis for clinicians. an updating article," Sao Paulo Medical Journal, vol. 138, no. 3, pp. 259-266, 2020.

[7] L. Magno, T. A. Rossi, F. W. d. Mendonça-Lima, C. C. d. Santos, G. B. Campos, L. M. Marques, M. Pereira, N. M. d. B. L. Prado, and I. Dourado, "Desafios e propostas para ampliação da testagem e diagnóstico para covid-19 no brasil," Ciência \& Saúde Coletiva, vol. 25, pp. 3355-3364, 2020.

[8] R. Caetano, A. B. Silva, A. C. C. M. Guedes, C. C. N. d. Paiva, G. d. R. Ribeiro, D. L. Santos, and R. M. d. Silva, "Desafios e oportunidades para telessaúde em tempos da pandemia pela covid-19: uma reflexão sobre os espacos e iniciativas no contexto brasileiro," Cadernos de Saúde Pública, vol. 36, p. e00088920, 2020.

[9] G. Jain, D. Mittal, D. Thakur, and M. K. Mittal, "A deep learning approach to detect covid-19 coronavirus with x-ray images," Biocybernetics and biomedical engineering, vol. 40, no. 4, pp. 1391-1405, 2020.

[10] R. Jain, M. Gupta, S. Taneja, and D. J. Hemanth, "Deep learning based detection and analysis of covid-19 on chest x-ray images," Applied Intelligence, vol. 51, no. 3, pp. 1690-1700, 2021.

[11] P. Endo, I. R. Silva, G. L. Santos, and D. Sadok, "Classifying covid-19 positive x-ray using deep learning models," IEEE Latin America Transactions, vol. 100, no. 1e, 2020.

[12] S. Basu, S. Mitra, and N. Saha, "Deep learning for screening covid-19 using chest x-ray images," in 2020 IEEE Symposium Series on Computational Intelligence (SSCI). IEEE, 2020, pp. 2521-2527.

[13] O. L. de Sousa, D. M. Magalhães, P. d. A. Vieira, and R. Silva, "Deep learning in image analysis for covid-19 diagnosis: a survey," IEEE Latin America Transactions, vol. 100, no. 1e, 2020.

[14] L. O. Hall, R. Paul, D. B. Goldgof, and G. M. Goldgof, "Finding covid-19 from chest x-rays using deep learning on a small dataset," arXiv preprint arXiv:2004.02060, 2020.

[15] M. M. A. Monshi, J. Poon, V. Chung, and F. M. Monshi, "Covidxraynet: Optimizing data augmentation and cnn hyperparameters for improved covid-19 detection from cxr," Computers in Biology and Medicine, vol. 133, p. 104375, 2021.

[16] C. Ouchicha, O. Ammor, and M. Meknassi, "Cvdnet: A novel deep learning architecture for detection of coronavirus (covid-19) from chest x-ray images," Chaos, Solitons 83 Fractals, vol. 140, p. 110245,2020 .
[17] C. Shorten and T. M. Khoshgoftaar, "A survey on image data augmentation for deep learning," Journal of Big Data, vol. 6, no. 1, pp. 1-48, 2019.

[18] L. Taylor and G. Nitschke, "Improving deep learning using generic data augmentation," arXiv preprint arXiv:1708.06020, 2017.

[19] L. Perez and J. Wang, "The effectiveness of data augmentation in image classification using deep learning," arXiv preprint arXiv:1712.04621, 2017.

[20] F. Chollet and J. Allaire, Deep Learning with R. Manning Publications, 2018. [Online]. Available: https://books.google. com.br/books?id=xnIRtAEACAAJ

[21] A. Sedik, A. M. Iliyasu, A. El-Rahiem, M. E. Abdel Samea, A. Abdel-Raheem, M. Hammad, J. Peng, A. El-Samie, E. Fathi, A. A. A. El-Latif et al., "Deploying machine and deep learning models for efficient data-augmented detection of covid-19 infections," Viruses, vol. 12, no. 7, p. 769, 2020.

[22] A. Waheed, M. Goyal, D. Gupta, A. Khanna, F. Al-Turjman, and P. R. Pinheiro, "Covidgan: data augmentation using auxiliary classifier gan for improved covid-19 detection," Ieee Access, vol. 8, pp. 91 916-91 923, 2020.

[23] F. Chollet, Deep Learning with Python. Manning, Nov. 2017.

$[24]$ A. L. C. Ottoni and M. Silva Novo, "A deep learning approach to vegetation images recognition in buildings: a hyperparameter tuning case study," IEEE Latin America Transactions, vol. 19, no. 12 , pp. 2062-2070, 2021.

[25] I. N. d. Silva, D. H. Spatti, and R. A. Flauzino, Redes neurais artificiais para engenharia e ciências aplicadas. Artliber Editora, 2010.

[26] S. Russell and P. Norvig, Artificial Intelligence: A Modern Approach. CreateSpace Independent Publishing Platform, 2016. [Online]. Available: https://books.google.com.br/books? id=PQI7vgAACAAJ

[27] C. Aggarwal, Neural Networks and Deep Learning: A Textbook. Springer International Publishing, 2018. [Online]. Available: https://books.google.com.br/books?id=achqDwAAQBAJ

[28] D. V. Minatto, S. Coral, and M. C. de Mattos Garcia, "Análise de características em redes neurais artificiais aplicadas em visão computacional com ênfase em reconhecimento facial," Anais SULCOMP, vol. 8, 2017.

[29] S. Peixoto, G. Cámara-Chávez, D. Menotti, G. Gonçalves, and W. Schwartz, "Brazilian license plate character recognition using deep learning," in Proceedings of the XI Workshop de Visão Computacional-WVC, 2015.

[30] C. I. Shimabukuro, N. V. Galegale, M. T. Okano, and C. Langhi, "Deep learing aplicado à negociação de ações por algoritmos: Uma revisão descritiva da literatura," South American Development Society Journal, vol. 6, no. 17, p. 237, 2020.

[31] A. M. Moura, "Redes neurais recorrentes aplicadas à previsão de preços de ativos da bovespa: comparativo de modelos para regressão," Matemática Bacharelado-Unisul Virtual, 2019.

[32] T. Okuyama, T. Gonsalves, and J. Upadhay, "Autonomous driving system based on deep q learnig," in 2018 International Conference on Intelligent Autonomous Systems (ICoIAS). IEEE, 2018, pp. 201-205.

[33] M. G. Bechtel, E. McEllhiney, M. Kim, and H. Yun, "Deeppicar: A low-cost deep neural network-based autonomous car," in 2018 IEEE 24th International Conference on Embedded and RealTime Computing Systems and Applications (RTCSA). IEEE, 2018, pp. 11-21.

[34] M. Elgendy, Deep Learning for Vision Systems. Manning Publications, 2020. [Online]. Available: https://books.google.pt/ books?id=97YCEAAAQBAJ

[35] A. Rosebrock, Deep Learning for Computer Vision with Python: Starter Bundle. PyImageSearch, 2017. [Online]. Available: https://books.google.com.br/books?id=9Ul-tgEACAAJ

[36] H. Panwar, P. Gupta, M. K. Siddiqui, R. Morales-Menendez, and V. Singh, "Application of deep learning for fast detection of covid-19 in x-rays using ncovnet," Chaos, Solitons \& Fractals, vol. 138, p. 109944, 2020. 